\title{
Association Between Genetic Polymorphisms of MIR3142HG and the Risk of Steroid-induced Osteonecrosis of the Femoral Head in the Population of Northern China
}

\section{Tiantian Wang}

Inner Mongolia Medical University

Huiqiang Wu

Inner Mongolia Medical University

Tingting Liu

Inner Mongolia Medical University

\section{Mneghu Sun}

Inner Mongolia Medical University

\section{Feimeng An}

Inner Mongolia Medical University

\section{Qiumei Dong}

Inner Mongolia Medical University

Jianzhong Wang ( $\sim$ wangjianzhongWJ@163.com)

The 2th affiliated Hospital of Inner Mongolia University https://orcid.org/0000-0003-4031-1316

\section{Research article}

Keywords: Steroid-induced osteonecrosis of the femoral head (steroid-induced ONFH), MIR3142HG, Genetic polymorphisms, Case-control study

Posted Date: October 14th, 2020

DOI: https://doi.org/10.21203/rs.3.rs-90412/v1

License: (c) This work is licensed under a Creative Commons Attribution 4.0 International License. Read Full License

Version of Record: A version of this preprint was published at Public Health Genomics on November 17th, 2021. See the published version at https://doi.org/10.1159/000519577. 


\section{Abstract}

Background: Steroid-induced osteonecrosis of the femoral head (ONFH) is aseptic necrosis of the femoral head caused by glucocorticoid use. Once necrotic femoral head necrosis occurs, it irreversibly affects the quality of life seriously. Studies have shown that the susceptibility to steroid-induced ONFH is likely to be related to the variation of miRNA coding genes. Therefore, this study aimed was to investigate the effect of MIR3142HG on steroid-induced ONFH.

Methods: Agena MassARRAY was used to genotype MIR3142HG gene rs1582417, rs2431689, rs7727155 and rs17057846 in 199 patients and 506 healthy people. A genetic model and haplotype analysis were used to evaluate the relationship between the MIR3142HG polymorphism and the risk of steroid-induced ONFH. The odds ratio (OR) and 95\% confidence intervals (Cls) were obtained through logistic regression to assess the influence of gene polymorphisms on the occurrence of steroid-induced ONFH.

Results: The consequences show that $\mathrm{rs} 7727115(\mathrm{OR}=0.76, \mathrm{p}=0.036)$ is a protective factor, it could reduce the risk of steroid-induced ONFH, rs1582417(OR=1.28, p=0.041) could increase the risk of steroid-induced ONFH. Stratified analysis, according to each clinical index shows that MIR3142HG TC-CC genotype facilitated the risk of steroidinduced ONFH in male ( $p<0.05)$. In addition, rs2431689 is related to HDL-C $(p=0.012)$ and ApoA1 $(p=0.010)$ levels, and rs17057846 ( $p=0.024)$ is related to ApoB levels. The linkage analysis indicated that three SNPs (rs2431689, rs7727115 and rs17057846) in MIR3142HG with significant chain imbalance. In addition, haplotype "GGG" of MIR3142HG was found out is harmful for steroid-induced ONFH.

Conclusion: Our results firstly confirm that the genetic polymorphism of MIR3142HG is associated with steroid-induced ONFH susceptibility in Chinese Han population.

\section{Introduction}

With the widespread, clinical use of hormone drugs, the reports of steroid-induced ONFH at home and abroad are gradually increasing. Steroid-induced ONFH is a serious concomitant hormone widely recognized in clinical practice disease, high disability rate, and seriously affect the physical and mental health of patients. In China, long-term or highdose corticosteroids are the most common cause of non-traumatic femoral head necrosis[1]. The pathogenesis is currently unclear, and studies have shown that the genome Changes in epigenetics are closely related to its occurrence and development, including genetic polymorphism and methylation changes.

Neuroimmune diseases and rheumatic diseases often need to be treated with glucocorticoids. Glucocorticoids can cause adverse reactions such as oedema, high sodium and low potassium, high blood sugar, centripetal obesity, and infection. Steroid-induced ONFH is the most common one of the severe adverse reactions. Abnormal glucocorticoid metabolism, transporter receptor and target, lipid metabolism disorder, coagulation and fibrinolysis dysfunction, inflammatory response and osteoporosis are the important pathogenesis of steroid-induced ONFH. Glucocorticoids act on osteoblasts, osteoclasts, and osteocytes, reducing fluid in the interstitial tubules of bone, blood supply and strength of bone, and causing damage [2]. However, not all glucocorticoids are used for treatment. All patients had femoral head necrosis, suggesting that there may be other risk factors or individual differences in the occurrence of steroid-induced ONFH. Current research has shown that the pathogenesis and genes of the disease SNPs are related to gene mutations. Steroid-induced ONFH may be a polygenic disease, such as lipid metabolism-related genes (ApoA, ApoB), drug metabolizing enzymes (CYP), drug transporters (ABCB1, ABCG2) and drug target molecules (NR3C1), etc.[3-5] For example, MMP-8, MMP-9, MMP-14 and TIMP4[6-9] have been confirmed to be associated with steroid-induced ONFH risk. 
MiRNAs are regulatory short - stranded non-coding RNAs found in eukaryotes.[9]. MiRNAs have been shown to participate in many biological and pathological processes, as well as tissue development[10]. The primary targeted pathways of miRNA with differential expression are mostly related to osteogenic differentiation, bone metabolism and vascular pathways. SNPs in miRNAs-encoding genes may alter the expression or function of their host miRNAs, thus associated with cancer-related risks [11-12].

To our knowledge, the contact between MIR3142HG polymorphism and the risk of steroid-induced ONFH has not been studied previously. Therefore, we designed a study to evaluate the MIR3142HG gene polymorphism at the allele, genotplotype and haplotype interface for the development of the steroid-induced ONFH in the population of northern China.

\section{Materials And Methods 2.1. Study Participants.}

From 2018 to 2020, a total of 705 subjects including 199 consecutive recruited steroid-induced ONFH and 506 healthy controls at the Second Affiliated Hospital of Inner Mongolia Medical University. The control is based on the physical examination enrollment group, without steroid-induced ONFH or other related diseases. This study was approved by the Hospital Ethics Committee. Informed consent of all participants.

All patients were diagnosed with hormonal femoral head necrosis in accordance with the relevant standards established by the International Bone Circulation Research Association. Inclusion criteria for the case group were longterm use of steroids, more than 16 mg per day or high-dose steroid impulsive treatment appears after more than 1 week; The patient presented with relevant clinical manifestations; such as hip pain, lower limb muscle atrophy, claudication, etc.[6] All outcome measures were confirmed as femur head necrosis by MRI X-ray bone scan or MRI. None of the patients had a history of alcohol consumption, chronic metabolic history, tumour history, hip trauma history or infection history. The patient had complete clinical data and no history of other treatments. Our exclusion criteria are patients who did not strictly meet the diagnostic criteria for hormonal cerebral haemorrhage and the diagnostic criteria for traumatic cerebral haemorrhage compared to other hip diseases; those with weekly alcohol intake of more than $400 \mathrm{ml}$ Patients. The healthy control group is defined according to the following criteria: No symptoms of hip-joint disease, no history of thromboembolism. People with severe chronic diseases and other significant family genetic diseases will be excluded.

\subsection{Genotyping assay}

In our study, blood samples of steroid-induced ONFH patients and normal control group were collected to analyze the difference of miR3142HG SNP in the blood of steroid-induced ONFH patients and normal control group and its correlation with clinical data, so as to study the application of miR3142HG in the diagnosis of steroid-induced ONFH. Rs1582417,rs2431689, rs7727115, and rs17057846 in the MIR3142HG gene were further selected. Blood samples were taken in EDTA tubes and centrifuged at $2000 \mathrm{rpm}$ for 10 minutes. Blood samples are stored at $-80{ }^{\circ} \mathrm{C}$ for future experiments. Genomic DNA was extracted from whole blood of steroid-induced ONFH patients and healthy controls, using the GoldMag-Mini Purification Kit (GoldMag Co. Ltd. Xian City, China). The genotyping of four SNPs (rs1582417, rs2431689, rs7727115,rs17057846) was put into practice on the Agena MassARRAY platform (San Diego, CA). And data management and analysis using the Sequenom TYPER software.

\subsection{Statistical Analyses.}


Results were presented as the mean \pm SD (standard deviation). All data were statistically analyzed using SPSS19.0. The difference in age between the two groups was analyzed by T-test or T 'test, and the functional analysis of SNP on SONFH susceptibility was performed by Logistic regression analysis. We used the Chi-squared test/Fisher's exact tests to compare the allelic and genotypic frequencies of cases and controls. ORs and $95 \% \mathrm{Cls}$ were determined using logistic regression analysis with adjustments for age and gender. Associations between the genotype polymorphisms and the risk of steroid-induced ONFH were evaluated under different genetic models using PLINK version 1.07 software. Finally, linkage disequilibrium among polymorphic sites was assessed with Haploview software package (version 4.2). With $P$ values $<0.05$ was considered statistically significant.

\section{Result}

A total of 705 people participated in this study of 199 patients ( 83 female, 116 male) and 506 healthy controls ( 83 female, 423 male). The mean ages were $42.58 \pm 13.154$ years for the cases and $41.21 \pm 12.900$ years for the controls. The information of the steroid-induced ONFH patients and healthy participants were shown in Table 1. There was no gender match between the patients and healthy participants in this study, and we will adjust this factor in the subsequent analysis.

Table 1

Characteristics of the participants

\begin{tabular}{|c|c|c|c|}
\hline Variables & Cases $(n=199)$ & Controls $(n=506)$ & $p$ value \\
\hline \multicolumn{4}{|l|}{ Sex $N$} \\
\hline Male & 116 & 423 & $<0.001^{\mathrm{a}}$ \\
\hline Female & 83 & 83 & \\
\hline Age, years (mean $\pm S D$ ) & $42.58 \pm 13.154$ & $41.21 \pm 12.900$ & $0.212^{b}$ \\
\hline \multicolumn{4}{|l|}{ Clinical stages } \\
\hline Stage I and II & 46 & & \\
\hline Stage III and IV & 153 & & \\
\hline \multicolumn{4}{|l|}{ Hip lesions } \\
\hline unilateral & 142 & & \\
\hline bilateral & 55 & & \\
\hline \multicolumn{4}{|c|}{ Course of disease(month) } \\
\hline More than 25 months & 67 & & \\
\hline 25 months or less & 132 & & \\
\hline \multicolumn{4}{|c|}{${ }^{a} p$ value was calculated by chi-squared test } \\
\hline \multicolumn{4}{|c|}{${ }^{b} p$ value was calculated by independent samples t test } \\
\hline$p<0.05$ indicates statis & significance & & \\
\hline
\end{tabular}


Four SNPs (rs1582417, rs2431689, rs7727115 and rs17057846) of MIR3142HG gene were selected for this casecontrol study. The basic information about all the SNPs, including alleles, MAF, OR, $95 \% \mathrm{Cl}$ and $\mathrm{HWE}$ results are presented in Table 2. It was found that rs $1582417(\mathrm{OR}=1.28,95 \% \mathrm{Cl}=1.01-1.62, p=0.041)$ were related to an increased risk of steroid-induced ONFH. On the other hand, $r s 7727115(\mathrm{OR}=0.76,95 \% \mathrm{Cl}=0.58-0.98, p=0.036)$ war related to a reduced risk of steroid-induced ONFH.

Table 2

Basic information of candidate SNPs of MIR3142HG gene in this study

\begin{tabular}{|c|c|c|c|c|c|c|}
\hline \multirow[t]{2}{*}{ SNP ID } & \multirow[t]{2}{*}{ Alleles A/B } & \multirow{2}{*}{\multicolumn{2}{|c|}{$\begin{array}{l}\text { MAF } \\
\text { Case Control }\end{array}$}} & \multirow[t]{2}{*}{ HWE-p } & \multirow[t]{2}{*}{ OR $(95 \% \mathrm{Cl})$} & \multirow[t]{2}{*}{$P$ value } \\
\hline & & & & & & \\
\hline rs1582417 & $\mathrm{C} / \mathrm{T}$ & 0.427 & 0.368 & 0.504 & $1.28(1.01-1.62)$ & $0.041^{*}$ \\
\hline rs2431689 & $A / G$ & 0.126 & 0.156 & 1.000 & $0.78(0.56-1.10)$ & 0.162 \\
\hline rs7727115 & $\mathrm{G} / \mathrm{T}$ & 0.260 & 0.317 & 0.918 & $0.76(0.58-0.98)$ & $0.036^{*}$ \\
\hline rs17057846 & $A / G$ & 0.214 & 0.205 & 1.000 & $1.05(0.79-1.40)$ & 0.719 \\
\hline \multicolumn{7}{|c|}{$\begin{array}{l}\text { SNP single-nucleotide polymorphism, HWE Hardy-Weinberg equilibrium, OR odds ratio, 95\% Cl 95\% confidence } \\
\text { interval, MAF minor allele frequency }\end{array}$} \\
\hline \multicolumn{7}{|c|}{$p$ were calculated by chi-squared test } \\
\hline${ }^{*} p<0.05$ indic & statistical sigr & & & & & \\
\hline
\end{tabular}

Subsequently, genetic models were used to compare the relationship between SNP genotypes and steroid-induced ONFH risk. As illustrated in Table 3, rs1582417 in the MIR3142HG gene was showed an increased risk of steroidinduced $\mathrm{ONFH}$ in the dominant model(OR $=1.44,95 \% \mathrm{Cl}: 1.01-2.07, p=0.046)$. At the same time, rs 1582417 showed a negative effect on steroid-induced ONFH under the Log-additive model $1 \mathrm{OR}=1.28,95 \% \mathrm{Cl}: 1.00-1.64, p=0.049$ ). Moreover, rs7727115 was found to be associated with a reduced risk of steroid-induced ONFH under the Log-additive model ( OR $=0.75,95 \% \mathrm{Cl}: 0.57-0.99, p=0.039)$. 
Table 3

Genotypic model analysis of relationship between SNPs and steroid-induced ONFH.

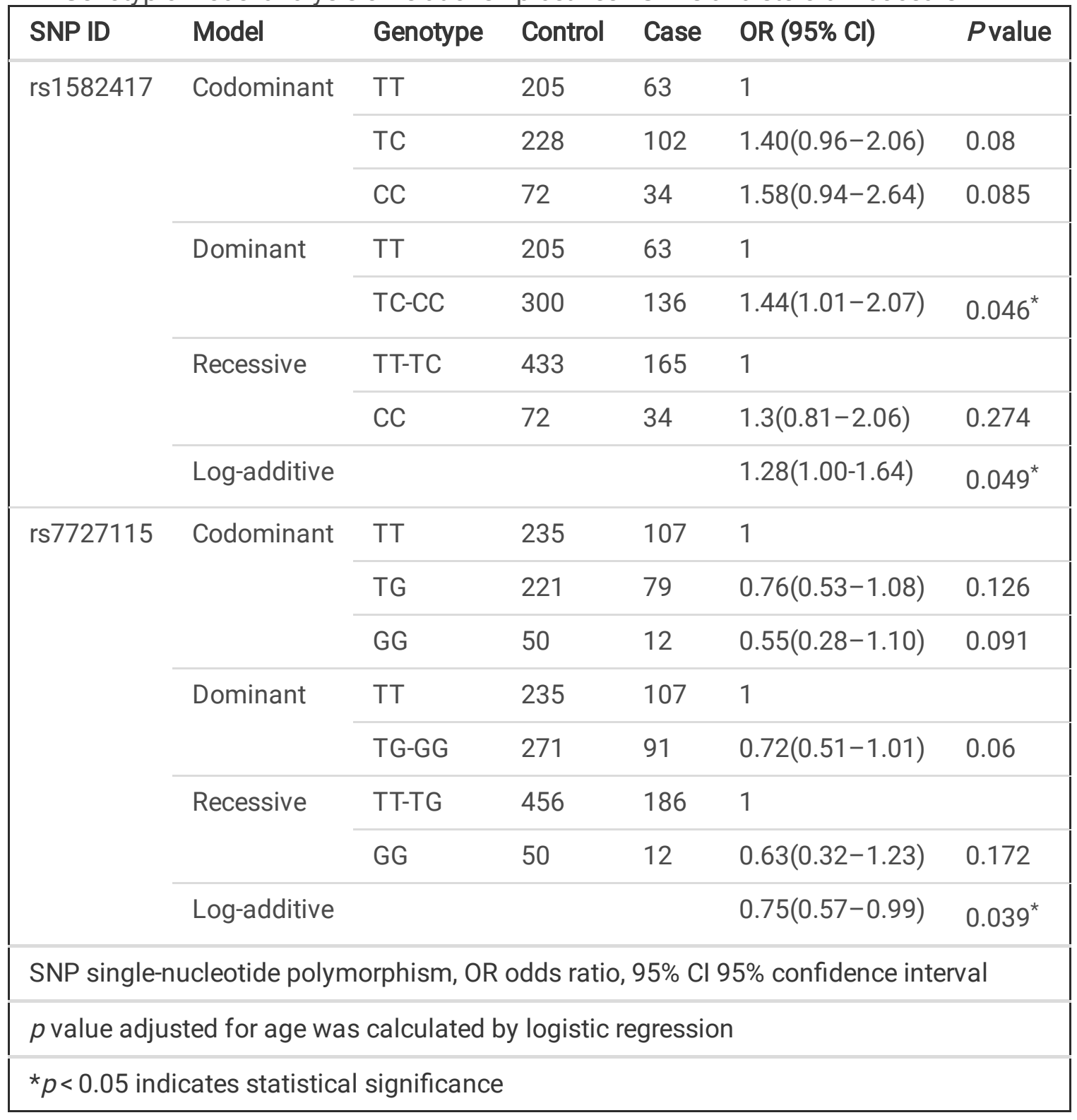

Stratified analysis by age, hip lesions classification and gender. The results show that in male group the MIR3142HG rs1582417 TC-CC genotype accelerated the risk of steroid-induced ONFH in the dominant model $(\mathrm{OR}=2.12,95 \% \mathrm{Cl}$ $1.15-3.36, p=0.001$ ) and in the codominant model risk of steroid-induced ONFH ( TC:OR $=2.09,95 \% \mathrm{Cl} 1.29-3.38, p=$ $0.012 ; \mathrm{CC}: \mathrm{OR}=2.28,95 \% \mathrm{Cl} 1.16-2.09, p=0.003)$. We observed that rs 17057846 shows association with unilateral and bilateral lesion of the hip joint $(p=0.049)$ (Table 4). In the Age(Older than 45), rs1582417 CC genotype and in the MIR3142HG gene are danger factors, increasing the risk of steroid-induced ONFH in the codominant model $(\mathrm{OR}=2.57$, $95 \% \mathrm{Cl} 1.13-5.86, p=0.024)$ and recessive model $(\mathrm{OR}=2.10,95 \% \mathrm{Cl} 1.03-4.32, p=0.043)$,log-additive model(OR= $1.59,95 \% \mathrm{Cl} 1.05-2.39, p=0.028)$.In addition, in the Age(Older than 45), rs7727115 TG genotype in the MIR3142HG gene are protect factors, reducing the risk of steroid-induced ONFH under the recessive model $(\mathrm{OR}=0.43,95 \% \mathrm{Cl} 0.23-$ $0.82, p=0.010)$ and dominant model TG-GG $(\mathrm{OR}=0.44,95 \% \mathrm{Cl} 0.24-0.81, p=0.008)$ and log-additive $\operatorname{model}(\mathrm{OR}=0.55$, $95 \% \mathrm{Cl} 0.33-0.90, p=0.019$ ). 
Table 4

Analysis of the relationship between MIR3142HG gene polymorphism and steroid-induced ONFH subgroups

\begin{tabular}{|c|c|c|c|c|c|c|c|}
\hline $\begin{array}{l}\text { Subgroup } \\
\text { analysis }\end{array}$ & SNP & Model & Genotype & Control & Case & OR (95\% Cl) & $\mathbf{p}$ \\
\hline \multirow[t]{8}{*}{ Male } & rs1582417 & \multirow[t]{3}{*}{ Codominant } & $\mathrm{TT}$ & 181(42.8\%) & $30(25.9 \%)$ & 1 & \\
\hline & & & $\mathrm{TC}$ & $182(43.0 \%)$ & $64(55.1 \%)$ & $\begin{array}{l}2.09(1.29- \\
3.38)\end{array}$ & $0.012^{*}$ \\
\hline & & & $\mathrm{CC}$ & $60(14.2 \%)$ & $22(19 \%)$ & $\begin{array}{l}2.28(1.19- \\
4.16)\end{array}$ & $0.003^{*}$ \\
\hline & & \multirow[t]{2}{*}{ Dominant } & $\mathrm{TT}$ & $181(42.8 \%)$ & $30(26 \%)$ & 1 & \\
\hline & & & TC-CC & $242(57.2 \%)$ & $86(74 \%)$ & $\begin{array}{l}2.12(1.34- \\
3.36)\end{array}$ & $0.001^{*}$ \\
\hline & & \multirow[t]{2}{*}{ Recessive } & TT-TC & $363(85.8 \%)$ & $94(81 \%)$ & 1 & \\
\hline & & & $\mathrm{CC}$ & $60(14.2 \%)$ & $22(19.0 \%)$ & $\begin{array}{l}1.44(0.84- \\
2.47)\end{array}$ & 0.187 \\
\hline & & Log-additive & $\ldots$ & $\ldots$ & $\ldots$ & $\begin{array}{l}1.55(1.16- \\
2.09)\end{array}$ & $0.330^{*}$ \\
\hline \multirow[t]{8}{*}{ Hip lesions } & rs17057846 & \multirow[t]{3}{*}{ Codominant } & GG & $81(57.0 \%)$ & $40(72.7 \%)$ & 1 & \\
\hline & & & GA & $55(38.7 \%)$ & $12(21.8 \%)$ & $\begin{array}{l}2.41(1.14- \\
5.09)\end{array}$ & $0.021^{*}$ \\
\hline & & & $A A$ & $6(4.2 \%)$ & $3(5.5 \%)$ & $\begin{array}{l}0.88(0.19- \\
4.04)\end{array}$ & 0.872 \\
\hline & & \multirow[t]{2}{*}{ Dominant } & GG & $81(57.0 \%)$ & $40(72.7 \%)$ & 1 & \\
\hline & & & GA-AA & $61(43 \%)$ & $15(27.3 \%)$ & $\begin{array}{l}2.11(1.05- \\
4.26)\end{array}$ & $0.037^{\star}$ \\
\hline & & \multirow[t]{2}{*}{ Recessive } & GG-GA & 136(95.8\%) & $52(94.5 \%)$ & 1 & \\
\hline & & & AA & $6(4.2 \%)$ & $3(5.5 \%)$ & $\begin{array}{l}0.65(0.15- \\
2.91)\end{array}$ & 0.574 \\
\hline & & Log-additive & $\ldots$ & $\cdots$ & $\cdots$ & $\begin{array}{l}1.63(0.89- \\
2.99)\end{array}$ & 0.111 \\
\hline \multirow[t]{5}{*}{$\begin{array}{l}\text { Age(Older than } \\
45)\end{array}$} & rs1582417 & \multirow[t]{3}{*}{ Codominant } & $\mathrm{TT}$ & $96(41.7 \%)$ & $19(27.1 \%)$ & 1 & \\
\hline & & & $\mathrm{TC}$ & $102(44.3 \%)$ & $34(48.6 \%)$ & $\begin{array}{l}1.42(0.73- \\
2.77)\end{array}$ & 0.305 \\
\hline & & & $\mathrm{CC}$ & $32(13.9 \%)$ & $17(24.3 \%)$ & $\begin{array}{l}2.57(1.13- \\
5.86)\end{array}$ & $0.024^{*}$ \\
\hline & & \multirow[t]{2}{*}{ Dominant } & TT & $96(41.7 \%)$ & $19(27.1 \%)$ & 1 & \\
\hline & & & TC-CC & 134(58.3\%) & $51(72.9 \%)$ & $\begin{array}{l}1.68(0.90- \\
3.14)\end{array}$ & 0.103 \\
\hline
\end{tabular}

$p$ values were calculated by logistic regression

${ }^{*} p<0.05$ indicates statistical significance 


\begin{tabular}{|c|c|c|c|c|c|c|c|}
\hline $\begin{array}{l}\text { Subgroup } \\
\text { analysis }\end{array}$ & SNP & Model & Genotype & Control & Case & OR (95\% Cl) & $\mathbf{p}$ \\
\hline & & \multirow[t]{2}{*}{ Recessive } & TT-TC & 198(86.1\%) & $53(75.7 \%)$ & 1 & \\
\hline & & & $\mathrm{CC}$ & $32(13.9 \%)$ & $17(24.3 \%)$ & $\begin{array}{l}2.10(1.03- \\
4.32)\end{array}$ & $0.043^{*}$ \\
\hline & & Log-additive & & $\ldots$ & $\ldots$ & $\begin{array}{l}1.59(1.05- \\
2.39)\end{array}$ & $0.028^{*}$ \\
\hline & \multirow[t]{8}{*}{ rs7727115 } & \multirow[t]{3}{*}{ Codominant } & TT & 107(46.3\%) & $45(65.2 \%)$ & 1 & \\
\hline & & & TG & $103(44.6 \%)$ & $20(29.0 \%)$ & $\begin{array}{l}0.43(0.23- \\
0.82)\end{array}$ & $0.010^{\star}$ \\
\hline & & & GG & $21(9.1 \%)$ & $4(5.8 \%)$ & $\begin{array}{l}0.50(0.15- \\
1.63)\end{array}$ & 0.249 \\
\hline & & \multirow[t]{2}{*}{ Dominant } & TT & 107(46.3\%) & $45(65.2 \%)$ & 1 & \\
\hline & & & TG-GG & $124(53.7 \%)$ & $24(34.8 \%)$ & $\begin{array}{l}0.44(0.24- \\
0.81)\end{array}$ & $0.008^{*}$ \\
\hline & & \multirow[t]{2}{*}{ Recessive } & TT-TG & $110(90.9 \%)$ & $65(94.2 \%)$ & 1 & \\
\hline & & & GG & $21(9.1 \%)$ & $4(5.8 \%)$ & $\begin{array}{l}0.69(0.21- \\
2.22)\end{array}$ & 0.535 \\
\hline & & Log-additive & $\ldots$ & $\ldots$ & $\ldots$ & $\begin{array}{l}0.55(0.33- \\
0.90)\end{array}$ & $0.019^{\star}$ \\
\hline \multicolumn{8}{|c|}{$p$ values were calculated by logistic regression } \\
\hline${ }^{*} p<0.05$ in & tintions & ance & & & & & \\
\hline
\end{tabular}

Effect on lipid metabolism level in patients, we found that the HDL-C and ApoA1 levels of the AA genotype carriers in rs 2431689 were higher than the GG genotype carriers, and the ApoB ratio of the GG genotype carriers in rs 17057846 was significantly lower than that of the AA genes. For genotype carriers, TC level AA genotype is significantly lower than GG genotype carrier. (Table 5) 
Table 5

The association of genotypes in MIR3142HG genes with the clinical phenotypes

\begin{tabular}{|c|c|c|c|c|c|c|c|}
\hline SNP & Genotype & $\begin{array}{l}\mathrm{TC} \\
(\mathrm{mmol} / \mathrm{L})\end{array}$ & $\begin{array}{l}\text { TG } \\
\text { (mmol/L) }\end{array}$ & $\begin{array}{l}\mathrm{HDL}-\mathrm{C} \\
(\mathrm{mmol} / \mathrm{L})\end{array}$ & $\begin{array}{l}\text { LDL-C } \\
(\mathrm{mmol} / \mathrm{L})\end{array}$ & ApoA1(mmol/L) & $\mathrm{ApoB}(\mathrm{mmol} / \mathrm{L})$ \\
\hline \multirow[t]{4}{*}{ rs1582417 } & $\begin{array}{l}\mathrm{TT}(\mathrm{n}= \\
63)\end{array}$ & $\begin{array}{l}4.46 \pm \\
0.95\end{array}$ & $\begin{array}{l}2.04 \pm \\
1.71\end{array}$ & $\begin{array}{l}1.08 \pm \\
0.27\end{array}$ & $\begin{array}{l}2.54 \pm \\
0.79\end{array}$ & $1.24 \pm 0.21$ & $0.98 \pm 0.78$ \\
\hline & $\begin{array}{l}\operatorname{TC}(\mathrm{n}= \\
102)\end{array}$ & $\begin{array}{l}4.52 \pm \\
0.91\end{array}$ & $\begin{array}{l}1.74 \pm \\
1.35\end{array}$ & $\begin{array}{l}1.05 \pm \\
0.26\end{array}$ & $\begin{array}{l}2.69 \pm \\
0.76\end{array}$ & $1.23 \pm 0.25$ & $0.93 \pm 0.24$ \\
\hline & $\begin{array}{l}\operatorname{CC}(n= \\
34)\end{array}$ & $\begin{array}{l}4.54 \pm \\
0.91\end{array}$ & $\begin{array}{l}1.75 \pm \\
1.22\end{array}$ & $\begin{array}{l}1.09 \pm \\
0.30\end{array}$ & $\begin{array}{l}2.67 \pm \\
0.71\end{array}$ & $1.26 \pm 0.25$ & $0.98 \pm 0.42$ \\
\hline & $p$ & 0.897 & 0.416 & 0.726 & 0.485 & 0.868 & 0.736 \\
\hline \multirow[t]{4}{*}{ rs2431689 } & $\begin{array}{l}G G(n= \\
151)\end{array}$ & $\begin{array}{l}4.47 \pm \\
0.93\end{array}$ & $\begin{array}{l}1.89 \pm \\
1.54\end{array}$ & $\begin{array}{l}1.04 \pm \\
0.25\end{array}$ & $\begin{array}{l}2.62 \pm \\
0.76\end{array}$ & $1.21 \pm 0.22$ & $0.96 \pm 0.56$ \\
\hline & $\begin{array}{l}\mathrm{GA}(\mathrm{n}= \\
44)\end{array}$ & $\begin{array}{l}4.62 \pm \\
0.91\end{array}$ & $\begin{array}{l}1.67 \pm \\
1.16\end{array}$ & $\begin{array}{l}1.17 \pm \\
0.31\end{array}$ & $\begin{array}{l}2.69 \pm \\
0.880\end{array}$ & $1.33 \pm 0.27$ & $0.92 \pm 0.24$ \\
\hline & $\mathrm{AA}(\mathrm{n}=3)$ & $\begin{array}{l}5.18 \pm \\
0.28\end{array}$ & $\begin{array}{l}2.33 \pm \\
0.73\end{array}$ & $\begin{array}{l}1.05 \pm \\
0.15\end{array}$ & $\begin{array}{l}3.21 \pm \\
0.56\end{array}$ & $1.32 \pm 0.29$ & $1.13 \pm 0.01$ \\
\hline & $p$ & 0.288 & 0.580 & $0.012^{*}$ & 0.388 & $0.010^{\star}$ & 0.756 \\
\hline \multirow[t]{4}{*}{ rs7727115 } & $\begin{array}{l}\operatorname{TT}(n= \\
12)\end{array}$ & $\begin{array}{l}4.79 \pm \\
0.95\end{array}$ & $\begin{array}{l}2.81 \pm \\
2.54\end{array}$ & $\begin{array}{l}1.01 \pm \\
0.25\end{array}$ & $\begin{array}{l}2.66 \pm \\
0.87\end{array}$ & $1.24 \pm 0.24$ & $0.95 \pm 0.22$ \\
\hline & $\begin{array}{l}\mathrm{TG}(\mathrm{n}= \\
79)\end{array}$ & $\begin{array}{l}4.50 \pm \\
0.95\end{array}$ & $\begin{array}{l}1.76 \pm \\
1.38\end{array}$ & $\begin{array}{l}1.06 \pm \\
0.26\end{array}$ & $\begin{array}{l}2.67 \pm \\
0.78\end{array}$ & $1.22 \pm 0.24$ & $0.90 \pm 0.24$ \\
\hline & $\begin{array}{l}\mathrm{GG}(\mathrm{n}= \\
107)\end{array}$ & $\begin{array}{l}4.47 \pm \\
0.90\end{array}$ & $\begin{array}{l}1.78 \pm \\
1.33\end{array}$ & $\begin{array}{l}1.08 \pm \\
0.28\end{array}$ & $\begin{array}{l}2.61 \pm \\
0.75\end{array}$ & $1.26 \pm 0.23$ & $0.99 \pm 0.65$ \\
\hline & $p$ & 0.542 & 0.056 & 0.699 & 0.838 & 0.472 & 0.515 \\
\hline \multirow[t]{4}{*}{ rs17057846 } & $\begin{array}{l}\mathrm{GG}(\mathrm{n}= \\
123)\end{array}$ & $\begin{array}{l}4.52 \pm \\
0.88\end{array}$ & $\begin{array}{l}1.88 \pm \\
1.53\end{array}$ & $\begin{array}{l}1.07 \pm \\
0.28\end{array}$ & $\begin{array}{l}2.66 \pm \\
0.74\end{array}$ & $1.24 \pm 0.26$ & $0.93 \pm 0.29$ \\
\hline & $\begin{array}{l}\mathrm{GA}(\mathrm{n}= \\
67)\end{array}$ & $\begin{array}{l}4.56 \pm \\
1.02\end{array}$ & $\begin{array}{l}1.80 \pm \\
1.35\end{array}$ & $\begin{array}{l}1.05 \pm \\
0.25\end{array}$ & $\begin{array}{l}2.67 \pm \\
0.81\end{array}$ & $1.23 \pm 0.19$ & $0.95 \pm 0.26$ \\
\hline & $\mathrm{AA}(\mathrm{n}=9)$ & $\begin{array}{l}3.93 \pm \\
0.45\end{array}$ & $\begin{array}{l}1.52 \pm \\
1.24\end{array}$ & $\begin{array}{l}1.16 \pm \\
0.27\end{array}$ & $\begin{array}{l}2.10 \pm \\
0.44\end{array}$ & $1.33 \pm 0.25$ & $1.40 \pm 2.03$ \\
\hline & $p$ & 0.151 & 0.742 & 0.518 & 0.094 & 0.495 & $0.024^{\star}$ \\
\hline \multicolumn{8}{|c|}{$p$ values were calculated by logistic regression } \\
\hline${ }^{*} p<0.05$ inc & es statisti & signific & & & & & \\
\hline
\end{tabular}


Table 6

The haplotype frequencies of MIR3142HG polymorphisms and their association with the risk of steroid-induced ONFH.

\begin{tabular}{|c|c|c|c|c|c|c|c|c|}
\hline & \multicolumn{3}{|l|}{ Haplotype } & \multirow[t]{2}{*}{ Freq } & \multicolumn{2}{|c|}{ Without adjustment } & \multicolumn{2}{|l|}{ With adjustment } \\
\hline & rs2431689 & rs7727115 & rs17057846 & & OR $(95 \% \mathrm{Cl})$ & $p^{a}$ & OR $(95 \% \mathrm{Cl})$ & $p^{b}$ \\
\hline 1 & $\mathrm{G}$ & G & $A$ & 0.2157 & $1.10(0.82-1.46)$ & 0.531 & $1.03(0.77-1.40)$ & 0.806 \\
\hline 2 & G & $\mathrm{T}$ & G & 0.2614 & $0.77(0.59-1.00)$ & 0.050 & $0.76(0.58-1.00)$ & 0.055 \\
\hline 3 & $A$ & G & G & 0.1269 & $0.82(0.58-1.16)$ & 0.267 & $0.81(0.57-1.16)$ & 0.244 \\
\hline 4 & G & G & G & 0.3959 & $1.33(1.04-1.70)$ & $0.024^{*}$ & $1.40(1.08-1.81)$ & $0.010^{*}$ \\
\hline \multicolumn{9}{|c|}{$p^{a}$ values were calculated by unconditional logistic regression. } \\
\hline \multicolumn{9}{|c|}{$p^{\mathrm{b}}$ values were calculated by unconditional logistic regression adjusted for age and gender. } \\
\hline \multicolumn{9}{|c|}{$p<0.05$ indicates statistical significance. } \\
\hline
\end{tabular}

In haplotype model analysis, one linkage disequilibrium (LD) block was detected in the MIR3142HG SNPs (rs2431689, rs7727115,rs17057846; Fig. 1). The "GGG" sequence was associated with an increased risk of steroid-induced ONFH $(\mathrm{OR}=1.33,95 \% \mathrm{Cl} 1.04-1.70, p=0.021$; adjusted $\mathrm{OR}=01.40,95 \% \mathrm{Cl} 1.08-1.81, p=0.010)$.

\section{Discussion}

Despite the introduction of many new treatment methods, and the rapid development of surgical techniques, the therapeutic effect of osteonecrosis has not improved significantly. Because many patients have reached the early stage of diagnosis, the final hip replacement is an inevitable choice. Therefore, researching the underlying mechanism of femoral head necrosis and development, and searching for more accurate biomarkers, are crucial for clinicians to formulate precise treatment strategies.

We assessed the relationship between the four SNPs in MIR3142HG and steroid-induced ONFH susceptibility in Han Chinese. The frequencies of 4 allelic SNPs of MIR3142HG genotype and haplotype in cases and control group were compared and further stratified. Among them, the rs 1582417 polymorphism is associated with increased risk of steroid-induced ONFH, while the rs7727115 mutation is associated with reduced risk. For all we know,this is the first case-control study to examine the relationship between MIR3142HG gene polymorphism and the risk of steroid-induced $\mathrm{ONFH}$. This research provides evidence for the biological significance of genetic variation in miRNA-coding genes, suggesting that it may affect the pathogenesis of steroid-induced ONFH.

MiRNA is highly conservative in evolution and its expression is tissue-specific. MiRNA has the following characteristics: Highly conserved; The order of time and space; Some MiRNAs are tissue-specific. Studies have shown that miRNAs play a crucial role in different physiological and pathological processes via regulating gene expression posttranscriptionally, that is, it specifically binds to the untranslated region (3 '-UTR) at the 3 ' -end of the mRNA of the target gene through base complementary pairing, inhibits the translation process of the mRNA or directly mediates the degradation of the mRNA, so as to achieve the purpose of regulating the expression of the target gene[13-15]. Recently, more and more miRNAs have been studied in orthopaedic diseases. Increasing evidence also indicates that miRNAs can directly play a role in the pathogenesis of many orthopedic diseases, regulating bone development and bone regeneration, such as disc degeneration, osteoarthritis, osteoporosis, and osteosarcoma.[16-19]. In recent years, 
MiRNAs have gradually become a research hotspot in the field of non-coding RNA, and some miRNAs have been proved to be closely related to the pathogenesis of steroid-induced ONFH.[20-21].

MIR3142HG, also known as the MIR146A host gene, is located on human chromosome 5q33.3. However, it is reported that MIR3142HG can produce miR-146a[22]. MiR-146a is abnormal in lung cancer, breast cancer, colorectal cancer, glioma and other cancers.[23-26]. MiR-146a may participate in ONFH and other physiological processes such as inflammation.[27].The study showed that MIR3142HG knockdown observably decreased the release of IL-8 and CCL2, and partially reduced the release of il-6 in control fibroblasts, suggesting that the decreased expression of MIR3142HG might be the reason for the decreased inflammatory reaction of IPF fibroblasts stimulated by IL-1[28]. It was found that MIR3142HG regulates the inflammatory response, and it is proved that the reduction of the inflammatory response of IPF fibroblasts is related to the decreased expression of MIR3142HG/miR-146a[28]. Yuan et al. designed a study to compare miRNA expression profiles in 9 ONFH patients and 6 femoral neck fractures. It was confirmed that the expression of Mir-146a was up-regulated in ONFH.[29]. So we doped out that MIR3142HG polymorphism might affect the expression or function of MiR-146a during the steroid-induced ONFH process. At present, the mechanism of MIR3142HG in steroid-induced ONFH has not been reported and remains to be studied.

We suggest that the induction age-related steroid-induced ONFH may relevance with rs1582417 and rs7727115 polymorphism in MIR3142HG. Further studies through gender stratification found that that rs1582417 polymorphism increases steroid-induced ONFH susceptibility, suggesting that the polymorphism risk association may be genderrelated. We found that the HDL-C and ApoA1 levels of the AA genotype carriers in rs2431689 were higher than the GG genotype carriers, and the ApoB ratio of the GG genotype carriers in rs17057846 was well below that of the AA genes. For genotype carriers, TC level AA genotype is significantly lower than GG genotype carrier. These results further illustrate the correlation between rs2431689, rs17057846, HDL-C levels, ApoA1 levels,ApoB levels, metabolic disorders, and osteonecrosis. At present, few functional studies have been reported. Understanding the function of miRNAs is crucial, which will provide new ideas for future treatment.

Some important findings were revealed in our study, but in interpreting these results, some limitations of this study should be considered. First, the sample size of hormonal femoral head necrosis is too small and lacks representativeness, while the large sample study takes time and effort; second, the relationship between genetic polymorphisms of different regions and races and the incidence of hormonal femoral head necrosis is inconsistent, and further analysis is needed. Reduce the variation within the interval and reduce the impact of the sampling error; again, the combination of large sample size aetiology and SNP analysis has become the direction of future research on steroid-related femoral head necrosis, but the credibility of the experimental results needs to be further improved, such as application of statistical methods such as multivariate analysis of variance and avoidance of confounding factors in case-control intervals.

\section{Conclusions}

In a word, This is the first evidence that two polymorphisms \rs1582417囚rs7727115) in MIR3142HG might contribute to the susceptibility to steroid-induced ONFH in the Chinese Han population. However, It is not clear whether these changes in MIR3142HG are consistent with the onset of ONFH, or simply reflect the terminal stage of the disease.

\section{Declarations}

\section{Acknowledgements}


We thank the patients who participated in this study and the participants in the control group. We would also like to thank all those who contributed to the collection of samples and analysis of data for this study.

\section{References}

1. Zhao Jie,Ma Xin-Long,Ma Jian-Xiong et al. TET3 Mediates Alterations in the Epigenetic Marker 5hmC and Akt pathway in Steroid-Associated Osteonecrosis.[J] .J. Bone Miner. Res., 2017, 32: 319-332.

2. Weinstein Robert S,Glucocorticoid-induced osteonecrosis.[J] .Endocrine, 2012, 41: 183-90.

3. Hirata Tetsurou,Fujioka Mikihiro,Takahashi Kenji A et al. Low molecular weight phenotype of Apo(a) is a risk factor of corticosteroid-induced osteonecrosis of the femoral head after renal transplant.[J] .J. Rheumatol., 2007, 34: 51622.

4. Hirata Tetsurou,Fujioka Mikihiro,Takahashi Kenji A et al. ApoB C7623T polymorphism predicts risk for steroidinduced osteonecrosis of the femoral head after renal transplantation.[J] .J Orthop Sci, 2007, 12: 199-206.

5. Kuribayashi Masaaki,Fujioka Mikihiro,Takahashi Kenji A et al. Combination analysis of three polymorphisms for predicting the risk for steroid-induced osteonecrosis of the femoral head.[J] .J Orthop Sci, 2008, 13: $297-303$.

6. Du Jieli,Jin Tianbo,Cao Yuju et al. Association between genetic polymorphisms of MMP8 and the risk of steroidinduced osteonecrosis of the femoral head in the population of northern China.[J] .Medicine (Baltimore), 2016, 95: e4794.

7. Du Jieli,Liu Wanlin,Jin Tianbo et al. A single-nucleotide polymorphism in MMP9 is associated with decreased risk of steroid-induced osteonecrosis of the femoral head.[J] .Oncotarget, 2016, 7: 68434-68441.

8. Qi Yuxin,Wang Jiaqi,Sun Mingqi et al. MMP-14 single-nucleotide polymorphisms are related to steroid-induced osteonecrosis of the femoral head in the population of northern China.[J] .Mol Genet Genomic Med, 2019, 7: e00519.

9. Mendell Joshua T,Olson Eric N,MicroRNAs in stress signaling and human disease.[J] .Cell, 2012, 148: $1172-87$.

10. Ambros Victor,The functions of animal microRNAs.[J] .Nature, 2004, 431: 350-5.

11. Króliczewski Jarosław,Sobolewska Aleksandra,Lejnowski Dawid et al. microRNA single polynucleotide polymorphism influences on microRNA biogenesis and mRNA target specificity.[J] .Gene, 2018, 640: 66-72.

12. Preskill Carina,Weidhaas Joanne B,SNPs in microRNA binding sites as prognostic and predictive cancer biomarkers.[J] .Crit Rev Oncog, 2013, 18: 327-40.

13. Li Zheng,Yu Xin,Shen Jianxiong et al. MicroRNA in intervertebral disc degeneration.[J] .Cell Prolif., 2015, 48: 27883.

14. Wang Yuan,Jia Lian-Shun,Yuan Wen et al. Low miR-34a and miR-192 are associated with unfavorable prognosis in patients suffering from osteosarcoma.[J] .Am J Transl Res, 2015, 7: 111-9.

15. Lv Cai,Bai Zhiming,Liu Zhenxiang et al. MicroRNA-495 suppresses human renal cell carcinoma malignancy by targeting SATB1.[J] .Am J Transl Res, 2015, 7: 1992-9.

16. Jing Wanli,Jiang Wenxue,MicroRNA-93 regulates collagen loss by targeting MMP3 in human nucleus pulposus cells.[J] .Cell Prolif., 2015, 48: 284-92.

17. Jackson Miriam T,Moradi Babak,Smith Margaret $M$ et al. Activation of matrix metalloproteinases 2,9 , and 13 by activated protein $C$ in human osteoarthritic cartilage chondrocytes.[J] ., 2014, 66: 1525-36.

18. Li Zheng,Shen Jianxiong,Chan Matthew T V et al. MicroRNA-379 suppresses osteosarcoma progression by targeting PDK1.[J] .J. Cell. Mol. Med., 2017, 21: 315-323. 
19. He Xiaoning,Zhang Wenkai,Liao Li et al. Identification and characterization of microRNAs by high through-put sequencing in mesenchymal stem cells and bone tissue from mice of age-related osteoporosis.[J] .PLoS ONE, 2013, 8: e71895.

20. Hao Cheng,Yang Shuhua,Xu Weihua et al. MiR-708 promotes steroid-induced osteonecrosis of femoral head, suppresses osteogenic differentiation by targeting SMAD3.[J] .Sci Rep, 2016, 6: 22599.

21. Gu Chenxi,Xu Yan,Zhang Shanfeng et al. miR-27a attenuates adipogenesis and promotes osteogenesis in steroidinduced rat BMSCs by targeting PPARY and GREM1.[J] .Sci Rep, 2016, 6: 38491.

22. Paterson Mark R,Kriegel Alison J,MiR-146a/b: a family with shared seeds and different roots.[J] .Physiol. Genomics, 2017, 49: 243-252.

23. Iacona Joseph R,Lutz Carol S,miR-146a-5p: Expression, regulation, and functions in cancer.[J] Wiley Interdiscip Rev RNA, 2019, 10: e1533.

24. lacona Joseph R,Monteleone Nicholas J,Lutz Carol S,miR-146a suppresses 5-lipoxygenase activating protein (FLAP) expression and Leukotriene B4 production in lung cancer cells.[J] .Oncotarget, 2018, 9: 26751-26769.

25. Si Chengshuai,Yu Qiao,Yao Yufeng,Effect of miR-146a-5p on proliferation and metastasis of triple-negative breast cancer via regulation of SOX5.[J] .Exp Ther Med, 2018, 15: 4515-4521.

26. Bleau Anne-Marie,Redrado Miriam,Nistal-Villan Estanislao et al. miR-146a targets c-met and abolishes colorectal cancer liver metastasis.[J] .Cancer Lett., 2018, 414: 257-267.

27. Guo Xiaoye,Zhang Ming,Li Qi et al. Evaluation of Genetic Variants in MIR3142HG in Susceptibility to and Prognosis of Glioma.[J] .Am. J. Clin. Oncol., 2020, 43: 1-8.

28. Hadjicharalambous Marina R,Roux Benoit T,Feghali-Bostwick Carol A et al. Long Non-coding RNAs Are Central Regulators of the IL-1 $\beta$-Induced Inflammatory Response in Normal and Idiopathic Pulmonary Lung Fibroblasts.[J] .Front Immunol, 2018, 9: 2906.

29. Yuan Heng-feng,Von Roemeling Christina,Gao Hui-di et al. Analysis of altered microRNA expression profile in the reparative interface of the femoral head with osteonecrosis.[J] .Exp. Mol. Pathol., 2015, 98: 158-63.

\section{Figures}

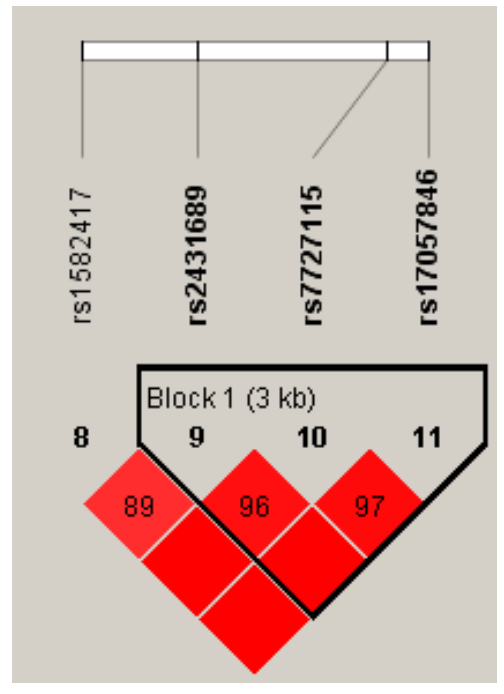

Figure 1 
Linkage disequilibrium (LD) analysis of the SNPs on MIR3142HG. Red squares display statistically significant associations between a pair of SNPs, as measured by D'; darker shades of red indicate higher D'. 